Chapter 9

\title{
Routes and requirements for realizing pervasive medical devices
}

Manuscript final revision v1_6, 10 April 2006

Book publication reference:

Craven M. P., Routes and requirements for realizing pervasive medical devices, Chapter 9, in Bardram J. E., Mihailidis A., Wan D. (Eds.), Pervasive Computing in Healthcare, CRC Press (Taylor \& Francis Group), November 2006. ISBN 084933621X.

Author and affiliation:

Dr. Michael P. Craven, Senior Research Fellow,

Multidisciplinary Assessment of Technology Centre for Healthcare (MATCH), Nottingham Hub

School of Electrical and Electronic Engineering

University of Nottingham, NG7 2RD.

Tel: $+44(0) 1159513804$

Fax: +44 (0)1159515616

Email: michael.craven @ nottingham.ac.uk

Biography :

Dr. Michael Craven is Senior Research Fellow at the University of Nottingham, engaged in healthcare research and industry liaison in the Multidisciplinary Assessment of Technology Centre for Healthcare. He has experience in electronic engineering and computer science teaching and research, including design of assistive and surgical simulation devices and software, and collaborative virtual environments. Current interests include methodologies for healthcare technology innovation and pervasive computing in healthcare. He obtained a BSc in Physics (1987) from the University of Bristol, an MSc in Modern Electronics (1989) and a PhD in the area of neural networks (1994), both from the University of Nottingham 
Summary

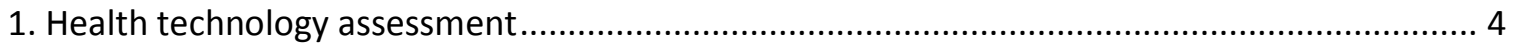

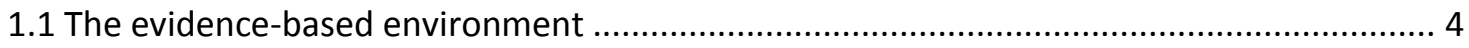

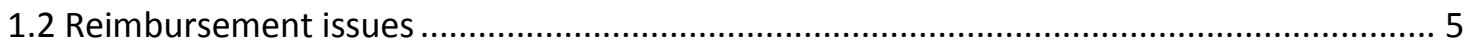

1.3 Health Technology and the role of HTA bodies .......................................................... 5

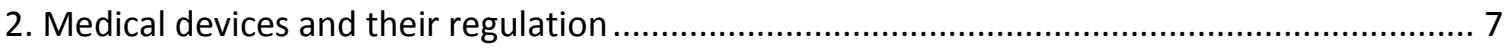

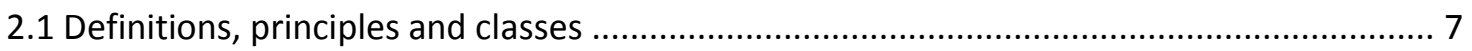

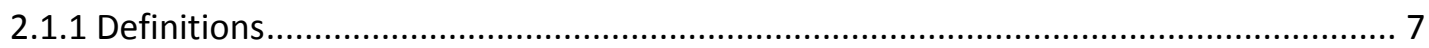

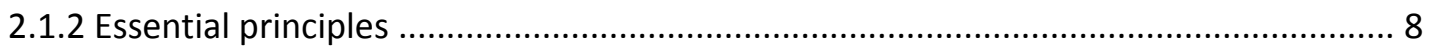

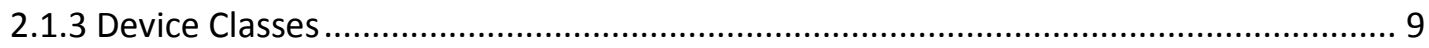

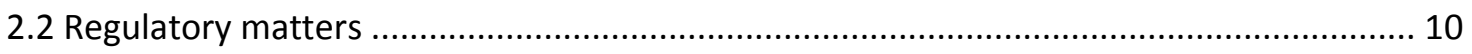

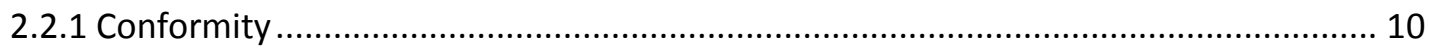

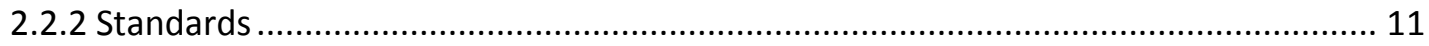

2.2.3 Post-market surveillance, vigilance and adverse incident reporting.......................... 12

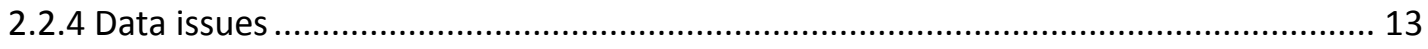

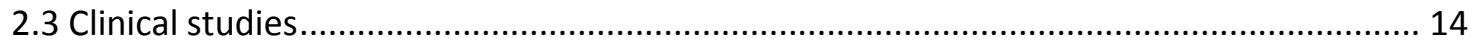

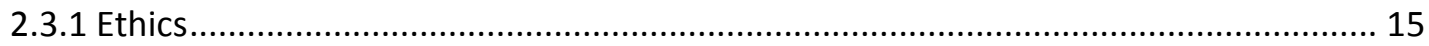

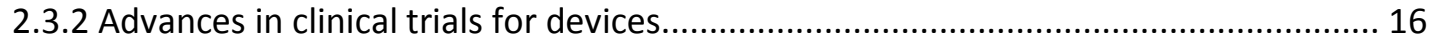

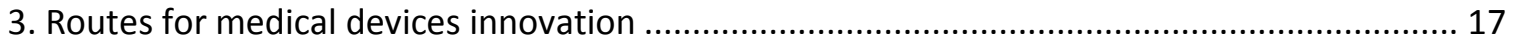

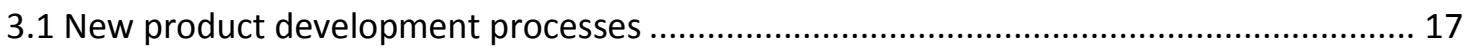

3.2 Special considerations for pervasive medical device development ................................. 18

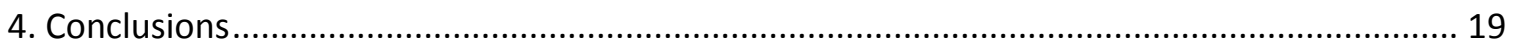

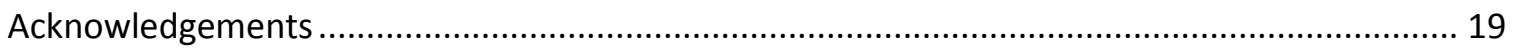

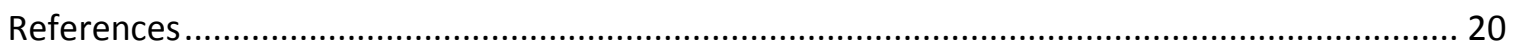

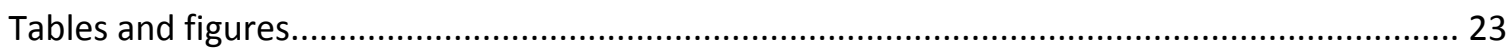




\section{Summary}

Pervasive computing brings together information and communications technology (ICT) through a wide variety of computing devices. Although most medical devices are not computing devices per se, a growing number of these are coming to rely on ICT whether by means of logging and telemetry functions (e.g. portable or home healthcare devices) or through their deployment in networked hospital environments. Furthermore, with the trend towards electronic patient records, it is likely that data from all manner of medical equipment will be transmitted through healthcare ICT systems. As a result of this convergence of technologies, pervasive healthcare design engineers need to have a good understanding of the regulatory environment in which medical device development and deployment is placed, and awareness of the evidence-based and cost-sensitive processes of healthcare technology assessment. This is on top of adherence to the increasingly sector-specific engineering methods and standards that guide electrical and computing equipment product designers in order to ensure safety-critical quality devices and systems.

This chapter aims to introduce the routes and requirements for realizing medical devices, enabling the pervasive healthcare engineer to enter the minds of the regulators and assessors and to better understand the industrial processes. In the first section the concept of Health Technology Assessment is introduced, the framework that judges the effectiveness and value of a device. The second section presents the regulations pertaining to medical devices in some detail including definitions and classifications, standards and quality systems, and the key aspects of conformity processes in Europe and North America. Some newly proposed concepts in clinical trials are outlined which may, if adopted, be particularly suited to medical devices. Also covered in this section are deployment and data issues, the former to ensure continued monitoring of the device and the latter arising from the link between the predominately independent worlds of devices and computer networks that are now coming together in pervasive healthcare. The third section examines the product design process for medical devices that is being developed by the Multidisciplinary Assessment of Technology Centre for Healthcare (MATCH) research programme. The concluding section reiterates the multidisciplinary environment of the pervasive healthcare innovator, and points to sources of information and advice. 


\section{Health technology assessment}

\subsection{The evidence-based environment}

Design engineers should already be intimately familiar with the concepts of product lifecycle, quality control standards and regulations, and increasingly with the need to take a user-centered approach. For successful healthcare technology development, however, we must include two additional concepts: firstly evidence-based practice, which began in clinical medicine but is now commonplace across healthcare including areas such as information management and device or facility design, and secondly an understanding of reimbursement processes for healthcare technologies. For the purposes of this chapter these two concepts are tied together into the process known as Health Technology Assessment (HTA).

Evidence-based practice is described by Booth as follows: ${ }^{1}$

'Evidence-based medicine (EBM) is "the conscientious, explicit, and judicious use of current best evidence in making decisions about the care of individual patients. The practice of evidence based medicine means integrating individual clinical expertise with the best available external clinical evidence from systematic research" (Sackett et al., 1996). Evidence-based practice is, by implication, the systematic application of rigorous scientific methods to the evaluation of the effectiveness of health care interventions. This can be broadened to include such considerations as appropriateness, clinical decision-making, economic evaluation, health technology assessment, outcomes measurement and risk management.'

The entrance of evidence-based practice in the context of healthcare technologies means not only that a new design should show efficacy in relation to risk for a specific therapeutic procedure by the gathering of appropriate clinical evidence (which may be mandatory, depending on the device class), but that it must prove its clinical effectiveness with respect to existing alternatives in terms of both efficacy and cost, and according to the healthcare allocation policy of the payer (e.g. equitable provision, or otherwise, of a treatment). Therefore evidence-based practice as related to healthcare technologies can be regarded to a great extent as the antithesis of the technology-push mentality found in the marketing of consumer electronics.

The implication for design engineers is that knowledge of its future assessment should feed into the development process of a device or system at as early a stage as possible, so that the appropriate design choices are made. As Baxter shows, early investment in the front-end of the design process is an important general principle for producing any successful new product, such that $85 \%-90 \%$ of its overall costs will have already been committed by decisions made before detailed design or production engineering begins. ${ }^{2}$ This figure may be even greater for medical devices because modifying a design at a late stage means retracing expensive and time-consuming regulatory processes. 


\subsection{Reimbursement issues}

Most medical technology products are quite different from consumer products since very few are available over-the-counter, and patients do not often pay the full cost of a device-related medical procedure or diagnostic test themselves. Instead, a medical device manufacturer will seek reimbursement from the patient's healthcare provider, who may be a public institution like a national health service or a private body such as a private clinic or health insurer. Reimbursement mechanisms vary greatly between different countries (and even between regions within some countries) and are quite complex in terms of the different mixes of public and private systems and product types covered. Access to specific healthcare products, especially in private care regimes, may also depend on patient factors such as age, income and employment status. From the technology manufacturer's point of view, this presents an unhelpfully fragmented and non-harmonized environment to market their products in.

As highlighted in a comprehensive Clinica report on reimbursement by Bromley et al., devices are being heavily implicated as a major cost driver for healthcare, and clinical evidence is increasingly being called for by the payers to justify these costs, whether it is for large capital items used for diagnostics or personal devices. ${ }^{3}$ Papatheofanis outlines five economic variables that payers typically use for health economic analysis: cost of therapy, cost of side-effects, costs avoided as a result of treatment, costs utilized based on information from diagnostics or referral of a patient, and costs utilized or saved during extended years of life as a result of therapy. ${ }^{4}$ For the design engineer, this necessitates some understanding of both treatment and funding pathways that a device will be involved in, and of the decision-making processes involved in having a device accepted for reimbursement. Detailed coverage of reimbursement methods is beyond the scope of this chapter, but it is worth mentioning the growth in popularity of a formal method known as Diagnostic Related Groups (DRGs) that was introduced by the major US health insurer Medicare and now has variants in many countries of the EU, Japan and Australia. DRGs are a classification of hospital case types into groups expected to have similar hospital resource use, which code for a tariff that is calculated from a fixed base rate along with a weighting to adjust for hospital size and other factors. An important characteristic of the DRG method of reimbursement is that it is not based on the cost of a device alone, but rather by the entire cost of the hospital resources made up by the clinical interventions and services required for diagnosis and treatment of a disease, plus its possible complications or comorbidities. Tariffs are intended to be updated on a regular basis (at least every few years) so that they represent the true cost of the procedure. DRGs introduce a high degree of transparency into the reimbursement process, but their existence makes it all the more important to predict how a device will map to all of its intended uses with their various reimbursement codes and to use this knowledge to inform early decision-making.

\subsection{Health Technology and the role of HTA bodies}

Health Technology Assessment (HTA) aims to provide information to support healthcare decisions and policy making at local, national and international levels. The University of York's Centre for Reviews and Dissemination (CRD) maintains an international database on behalf of the International Network of Agencies for Health Technology Assessment including a list of HTA bodies worldwide and records of 
ongoing projects being conducted. ${ }^{5}$ One example of an HTA body, the UK's National Health Service (NHS) R\&D division HTA programme, usefully defines health technology as follows: ${ }^{6}$

'Health Technology is an internationally recognised term that covers any method used to promote health, prevent and treat disease and improve rehabilitation or long-term care. 'Technologies' in this context are not confined to new drugs or pieces of sophisticated equipment, but include procedures, settings of care and screening programmes.'

Many HTA bodies are closely involved with reimbursement and the economics of healthcare delivery and they provide a speed of review that is faster when compared to the time required for rigorous surveys of clinical evidence, and in practice draw on existing surveys. The use of HTA in practice is typified by its fundamental role in the National Institute of Clinical Excellence (NICE) of England and Wales that was established in 1999 to appraise appropriate use of both new and existing health technologies, which include pharmaceuticals, medical devices, diagnostic techniques, clinical procedures and health promotion. ${ }^{7}$ Each NICE appraisal invites evidence from stakeholders including professionals, patient groups and manufacturers. Alongside this a Technology Assessment Report on clinical and costeffectiveness is commissioned from the national HTA Programme. NICE then uses the combined information from reports and stakeholders to inform its policy-making decisions. HTA gives government organizations like NICE considerable power over the introductions of new technologies and removal of existing ones if they become to be regarded as clinically ineffective or too costly. Therefore its growing prevalence and influence is something that healthcare technology developers should be aware of. The next section covers the equally important area of medical device regulation and standards. 


\section{Medical devices and their regulation}

\subsection{Definitions, principles and classes}

Medical devices are highly regulated. This is essential to ensure patient and practitioner safety, good performance and quality of manufacture in the pre-market stage, correct listing and advertising of the device that is being placed on the market, and fulfillment of post-market obligations such as detecting and alerting users of any problems and monitoring of a device's clinical performance. The following sections are intended to give the reader an introduction to the essential pre-market regulatory processes and some of the post-market obligations with respect to the planning and realization of a device.

\subsubsection{Definitions}

The first step in the regulatory process towards marketing a medical technology product is knowing that it is a medical device. Definitions and nomenclatures for medical devices are not internationally agreed although this is being worked on by the Global Harmonization Task Force (GHTF), which was founded in the 1992 by the European Union (EU), United States (US), Canada, Australia and Japan. ${ }^{8}$ These efforts have been facilitated by the passing of EU-wide harmonization legislation, the US Federal Drug Administration (FDA) Modernization Act, and through substantial adoption of GHTF recommended models by the Therapeutic Goods Administration (TGA) in Australia, the Therapeutic Products Directorate (TPD) in Canada, and by Japan's Ministry of Health, Labor, and Welfare (MHLW). Naming of devices is becoming standardized via ISO 15225 Nomenclature - Specification for a nomenclature system for medical devices for the purposes of regulatory data exchange, which is helping to facilitate international consensus on a Global Medical Device Nomenclature (GMDN) that is endorsed by the GHTF. The GHTF has proposed a harmonized definition for medical devices (document SG1/N029R11), ${ }^{8}$ but it is instructive to see the differences between the US and EU as they currently stand. Therefore, definitions for medical devices in the two regions will now be considered.

The FDA, via its Center for Devices \& Radiological Health, describes a medical device as: ${ }^{9}$

'an instrument, apparatus, implement, machine, contrivance, implant, in vitro reagent, or other similar or related article, including a component part, or accessory which is:

- recognized in the official National Formulary, or the United States Pharmacopoeia, or any supplement to them,

- intended for use in the diagnosis of disease or other conditions, or in the cure, mitigation, treatment, or prevention of disease, in man or other animals, or

- intended to affect the structure or any function of the body of man or other animals, 
and which does not achieve any of it's primary intended purposes through chemical action within or on the body of man or other animals and which is not dependent upon being metabolized for the achievement of any of its primary intended purposes.'

Within the EU, medical devices are covered by three important pieces of legislation comprising of the Medical Devices Directive (MDD), the Active Implantable Medical Devices Directive (AIMDD) and the In Vitro Diagnostics Directive (IVDD). The AIMDD applies to all active devices and related accessories intended to be permanently implanted in humans, the IVDD applies to all devices and kits used away from the patient to make a diagnosis of patient medical conditions, and the MDD covers all other devices that are not in the two special categories. Non-device products on the other hand are covered by a directive known as the Community Code on Medicinal Products for Human use.

The MDD (93/42/EEC) defines a medical device as: ${ }^{10}$

'any instrument, apparatus, appliance, material or other article, whether used alone or in combination, including the software necessary for its proper application, intended by the manufacturer to be used for human beings for the purposes of:

- diagnosis, prevention, monitoring, treatment or alleviation of disease,

- diagnosis, monitoring, treatment or alleviation of or compensation for an injury or handicap,

- investigation, replacement or modification of the anatomy or of a physiological process,

- control of conception,

and which does not achieve its principal intended action in or on the human body by pharmacological, immunological or metabolic means, but which may be assisted in its function by such means.'

Part of the rationale behind the form of these definitions is to distinguish devices from pharmaceutical products. Although from a patient's point of view, devices and drugs are complementary parts of managing their health, there are some important differences from $\mathrm{HTA}^{7}$ and other perspectives as shown in Table 1. Within MDD definitions an infusion pump that supplies a pharmaceutical is a device whereas a conventional pill capsule is not, and some drug-device combinations are also covered as devices. It is worth noting that some recent developments such as biologic coatings, tissue-engineered products and other drug-device combinations are further pushing at the borderline, so that clarifications to legislation have been and continue to be necessary.

(INSERT TABLE 1 ABOUT HERE - find table after references)

\subsubsection{Essential principles}

In addition to definitions, the MDD provides Minimum Essential Requirements (Annex I) for the design and manufacture of medical devices to ensure the protection of the health and safety of patients, users and 
third parties, which makes safety a central principle in the legislation ${ }^{11}$. The requirements include (although this list is not exhaustive):

- a general requirement for inherently safe design and construction,

- safety and compatibility of materials, including medicines if acting as an ancillary device,

- no adverse effect on characteristics or performance from normal use, transportation or storage,

- elimination, minimization, protection against and informing of risk, ensuring residual risk is acceptable when weighed against benefit to the patient. Risks include those posed by:

- substances leaking into or out of the device,

- infection and microbial contamination,

- tissues of animal origin,

$\circ$ ionizing radiation,

- a device being connected to, or equipped with, an energy source (electrical, mechanical, thermal risks),

- combination of devices with, and connection to, other systems,

$\circ$ environmental conditions, incl. interference with other devices, and,

$\circ$ aging of a device.

- ergonomic design,

- sufficient accuracy, if a measuring device, and,

- adequate product marking and user instructions.

The MDD essential requirements have for the most part been adopted by the GHTF Essential Principles of Safety \& Performance of Medical Devices (SG1-N02OR5) ${ }^{8}$ and are therefore likely to be the basis for future international consensus.

\subsubsection{Device Classes}

Once you know your product is a medical device, the next important step is to know its class since this determines the route to conformity. Again, although not universally agreed by regulators, there is a degree of harmonization whereby medical devices are classified into three or four classes according to 
level of risk, use and degree of invasiveness. The higher the risk, the higher the class number. Devices may also be classified during development e.g. the FDA classifies investigational devices in two classes: Significant Risk (SR) or Non-Significant Risk (NSR).

Currently, however, there are alternative approaches to actually determining the device class for a new device. In the EU this is done by applying a set of 18 rules (as specified in MDD Annex IX) with additional special rules for Active Implantable Medical devices and In Vitro Diagnostic devices, whereas in the US classification is carried out by government expert panels under Title 21 of the Code of Federal Regulations (CFR) whereby a device is generally assumed to be high risk unless it can be shown otherwise. The panel method can introduce uncertainty into the process, which can be especially worrying if the device is bordering on high risk since this increases the cost of obtaining conformity. On the other hand, a rulebased type method can be difficult to apply although for the MDD there are some useful additional guideline documents. ${ }^{12}$ Table 2 shows the types of devices in the various classes which result from the MDD rules. Non-active and accessory equipment is classified along with the active devices that are of most interest to pervasive healthcare designers. Further discussion about classification in the EU and US and global trends can be found in Davey et al. ${ }^{13}$

(INSERT TABLE 2 ABOUT HERE - find table after references)

\subsection{Regulatory matters}

\subsubsection{Conformity}

Conformity is the successful outcome of the compulsory regulatory processes by which a medical device will be accepted for marketing in a particular region. In the EU, conformity is shown by obtaining CE marking which means the device is certified to have met regulatory requirements. This includes adherence to the essential principles of the directives as assessed by an accredited third party (Notified Body) in the case of Class II/III devices or via self-assessment in the case of a Class I low risk device (provided the company is registered as meeting a quality systems management standard). In the UK, notified bodies are audited by the Medicines and Healthcare products Regulatory Agency (MHRA) of the Department of Health. In the US, conformity is shown by obtaining FDA approval from the government by going through one of two processes. Premarket approval (PMA) involves demonstration of reasonable safety and effectiveness which is required for a high-risk or new kind of device. A less-stringent Premarket Notification $510(\mathrm{k})$ process is allowed for devices that are substantially equivalent (SE) to a device already on the market, known as a predicate device. According to the FDA: ${ }^{9}$

'a device is deemed to be SE if, in comparison to a predicate device it: has the same intended use as the predicate device; and has the same technological characteristics as the predicate device; or has different technological characteristics, that do not raise new questions of safety and effectiveness, and the sponsor demonstrates that the device is as safe and effective as the legally marketed device. A claim of substantial equivalence does not mean the new and predicate devices must be identical. Substantial equivalence is established with respect to intended use, design, energy used or delivered, materials, performance, safety, effectiveness, labeling, biocompatibility, standards, and other applicable characteristics.' 
Some 1,700 devices are already classified by the FDA so it is essential for the innovator of a device to check for substantial equivalence in determining the likely route to compliance for marketing it in the US. An illustrative example of SE devices are 3D navigation systems for computer-aided orthopedic surgery which are predicated to stereotaxic equipment that was originally developed for neurosurgery. Because of this several manufacturers have obtained approval via the $510(\mathrm{k})$ route. In contrast, companies found it difficult to obtain FDA approval for robotic orthopedic surgery systems due in part to the lack of a suitable predicate device, which therefore necessitated the PMA route.

\subsubsection{Standards}

Adherence to product standards is a vital component of the regulatory process, and although pre-market standards vary in format in different regions, they universally apply to three main areas according to the World Health Organization: ${ }^{14}$

- Device attributes: Product safety and performance

- Manufacturing: Quality system

- Labeling: Description, and instructions for use

There are a large number of international and regional standards relating to medical devices ${ }^{15}$. For an overview of such standards, see ISO TR16142 Medical devices - Guidance on the selection of standards in support of recognized essential principles of safety and performance of medical devices. There is also a fairly recent comprehensive reference by Fries. ${ }^{16}$ Device attributes standards are very much related to the essence of the essential requirements as previously described for the MDD. Data standards, since they are very important to pervasive systems, will be considered later on in this section. Standards for clinical studies, although linked to safety and performance, will also be mentioned in a separate section. Manufacturing standards are for the most part covered by quality assurance (QA) systems that are required for the production and testing of all classes of device. In the EU this is now covered by the medical device standard ISO 13845. In the US, a company must follow Good Manufacturing Practice/Quality Systems (GMP/QS) standards. These standards require design controls and documentation for the manufacturing process. Labeling standards contribute to safe use and tracking of products.

Rather than go into specific details, it is perhaps more useful in a changing regulatory environment to examine trends in the evolution of standards in recent years, as follows (with relevant examples):

- Regulations are subsuming many voluntary standards, which is deemed useful because it is easier to change a standard than a regulation and this also aids governments in creating regulations. An exception is the area of environment protection which is becoming more regulated (see below).

- Standards are becoming more sector specific. For example, the general ISO 9001 quality standard is now for the most part incorporated into ISO 13485 Medical devices - Quality management systems - Requirements for regulatory purposes. ISO 90003 Software engineering - Guidelines for the application of ISO 9001:2000 to computer software and IEC 61508 Functional Safety of 
Electrical / Electronic / Programmable Electronic Safety-related Systems are likely to be supplemented by IEC 62304 Medical device software - software life-cycle processes.

- Standards are coming more internationally recognized e.g. updated US and Japanese GMP standards are being based on the new ISO 13485:2003. This is intended to assist in facilitating global trade and access to new technologies. The GHTF has also proposed a documentation system to harmonize collation of all the material needed for pre-market approval applications, known as Summary Technical Document (STED), which is currently being piloted and evaluated by its members.

- Standards are becoming less prescriptive in terms of tools and methods, but instead are taking on essential principles (e.g. for performance) and a risk management philosophy that requires the manufacturer to actively take the responsibility for implementing and continually reviewing its processes to analyze, identify and control risk. As an example, the forthcoming 3rd edition of IEC 60101 Medical Electrical Equipment will require adherence to ISO 14971 Medical devices application of risk management in medical devices.

- Usability engineering is becoming less of a guideline and more of a requirement. This is related to risk management and arose from the need to understand and mitigate potential misuse. In particular usability will be fully incorporated into the 3rd edition of IEC 60101 and the scope of use will also change from 'under medical supervision' to 'all use'. This is alongside recognition of the need to have tight controls on home use.

- Sustainability and environmental protection are becoming more regulated, building on voluntary environmental management standards such as ISO 14001. Example include the EU's Waste from Electrical \& Electronic Equipment (WEEE) and Restriction of Certain Hazardous Substances (RoHS) directives and Integrated Product Policy (IPP), where greater responsibility is placed on manufacturers regarding the disposal of devices, necessitating 'cradle-to-grave' engineering. The Eco-design requirements for energy-using products (EuP) directive is intended to make electrical devices more environmentally friendly. Japan has also passed a set of environmental laws including Home Appliances Recycling Law (HARL), Law for the Effective Utilization of Resources (LPEUR) and Green Purchasing Law (GPL).

- Recognition is rising regarding the need to ensure safety of refurbished and denoted equipment as well as the reprocessing of devices labeled for single-use. This includes equipment intended for reuse in developed countries, and, for trade and donation of equipment to developing countries, some of whom have previously had bad experiences with used equipment. ${ }^{14}$

\subsubsection{Post-market surveillance, vigilance and adverse incident reporting}

Once a device is on the market and is being used by practitioners and patients, it is important that its safety and performance continue to be assessed. Devices can be used and misused in many different and unforeseen ways (perhaps as a result of inadequate labeling and user instructions) and, in spite of premarket efforts, can fail in actual use or otherwise incur complaints from customers. Changes in clinical practice may also impact on actual use of a device, while advances in state-of-the-art technology can 
demand changes to a design. This is quite apart from the processes of HTA which as we have seen are used to assess and reassess clinical effectiveness and cost effectiveness for policy-making purposes.

The two main methods by which post-market assessment is implemented are post-market surveillance (PMS) studies by the manufacturer and by gathering and disseminating information through adverseincident reporting networks. In Europe a related term vigilance is used to denote the responsibility of the manufacturer to track and report on problems arising with a device.

Post-market activities are for the most part covered by the requirements of standards and regulations. For example, under the US Code of Federal Regulations (CFR) the FDA may impose post-market evaluation and periodic reporting on a manufacturer as part of the PMA approval of a device (21 CFR Part 814) and can also order subsequent postmarket surveillance for any Class II and III device where there is a serious risk to health from device failure, as well as for long-term implants or life-sustaining devices (21 CFR Part 822). The EU MDD requires the manufacturer to have systematic procedure to deal with post-market experience of a device. Additionally the relevant body (Competent Authority) must be informed of any deficiencies that resulted (or could have resulted) in death or serious deterioration of health and/or of the technical or medical reason for the recall of a device. There are also a variety of methods recommended for conducting PMS such as active supervision, customer surveys, inquiries of users and patients, literature reviews, and post-market clinical follow-up (PMCF). PMCF is recommended for capturing infrequent complications and long-term performance issues that would not be detected in the pre-market phase, as outlined in guidance MEDDEV $2.12 / 2 .^{12}$

\subsubsection{Data issues}

A key feature of pervasive healthcare is the interaction of devices with information and communication technology (ICT) systems. This convergence of technologies is becoming manifest by widespread use of commercial off-the-shelf (COTS) hardware and software such as the personal computer, and the use of standards-based communications such as wired and wireless Ethernet as outlined by Cohen. ${ }^{17}$ As Cohen points out, COTS and data communication technologies can together facilitate automatic data collection, analysis, reporting, dynamic reconfiguration and remote software upgrading for medical devices. The move to electronic patient records is likely to go hand in hand with increased demand for devices with a computer interface.

There are a number of standards and initiatives in the area of data communications that are specific to medical technologies. One such industry standard is Digital Imaging and Communication in Medicine (DICOM) which originates from the need to transfer medical images from computerized tomography (CT) and magnetic resonance imaging (MRI) scanners. The other well-established industry standard is High Level 7 (HL7) designed for hospital administrative data exchange. IEEE 1073/ISO 11073 Health informatics - Point-of-care medical device communication is an international standard that currently at the draft stage. A recent initiative emanating from the US is Integrating the Hospital Environment (IHE) which aims to bring together usage of DICOM, HL7 and internet protocols in healthcare systems within a set of Technical Frameworks for various healthcare domains e.g. IT Infrastructure, Cardiology, Laboratory, Radiology. ${ }^{18}$ Furthermore, the US National Academies have recently published an in-depth report highlighting the need for partnership between engineering and medicine in order to apply the best 
systems engineering approach to healthcare delivery that is, 'safe, effective, timely, patient-centered, efficient and equitable'. ${ }^{19}$

Data security is an issue that is receiving particular attention with the introduction of electronic patient records. In the US, the Health Insurance Portability and Accountability Act (HIPAA) requires, as of 21 April 2005 , that healthcare providers adopt a security procedure to ensure the integrity, availability and confidentiality of information maintained and transmitted by medical devices. ${ }^{20}$ From a service provision perspective, Grimes outlines the need to shift from management of discrete devices towards an overall policy of safeguarding information, and lists a range of device and system requirements that might be used to mitigate security threats. ${ }^{21}$ These threats include hardware failures or errors, user misuse or abuse of a device, erroneous data entry, malicious assault, unauthorized data access and/or modification, and environmental effects such as electromagnetic interference (EMI) or interruption of utilities such as power. These and other issues related to data handling and protection must therefore feed into the design process for pervasive healthcare devices and systems.

\subsection{Clinical studies}

A key aspect within the pre-market processes for proving and approving medical devices is the gathering and use of data concerning its clinical efficacy and safety. For certain classes of device, clinical approval resulting from the outcomes of testing on human subjects will be mandatory for its market certification. This might involve actual clinical studies or the compilation and critical analysis of existing evidence from the literature and other sources concerning similar devices. ${ }^{22}$ Even for low risk devices, clinical data may be important for proving the need and benefits of a product to users and payers.

Pre-market clinical studies typically take place after detailed design of the device is completed. Data which is intended to be used for predicting clinical effectiveness on living humans, especially for invasive devices, is also commonly obtained in the development stage from animal experiments, human cadavers and tissue. Pre-clinical information may also be gained from systematic consultation of individuals or groups of healthcare professionals and patients, as well as from modeling exercises.

If the conducting of a clinical trial is necessary due to insufficient existing evidence, the manufacturer is required to follow procedures in applying for permission to do so, and for the running of the trial. The processes for doing this are regulated by the same bodies as in section 2.2.1, and various standards are applicable. Devices at this stage are legislated for their clinical study in the US through the Investigative Device Exemption (IDE) regulation (21 CFR 812) and in the EU through clinical investigation requirements in the MDD, with guidance issued via MEDDEV 2.7 Clinical investigation, clinical evaluation: Guide for manufacturers and notified bodies. ${ }^{12}$ International standard ISO 14155 Clinical Investigation of Medical Devices for Human Subjects covers recognized practices for conducting trials. In the US, and elsewhere, clinical and laboratory practices are governed by requirements known as Good Clinical Practices (GCP) and Good Laboratory Practices (GLP).

Although the details of clinical trials methods and approval processes are outside the scope of this chapter, the remainder of the section will concentrate on ethical approval aspects since this is also relevant to pre-clinical studies that device designers may also be involved in. Some developments in post- 
market clinical trial design, that are intended to be more suitable for medical device evaluation from an HTA perspective, will also be introduced.

\subsubsection{Ethics}

Before conducting human clinical studies, and indeed even before systematic pre-clinical consultations with professional and patients (or healthy volunteers), it is a normal requirement to have obtained ethical approval from the institutions involved. This typically involves interaction with healthcare service providers. By way of an example, within the UK's National Health Service there are local research ethics committees (LRECS) who make decisions about single-centre studies and multi-site research ethics committess (MRECs) for streamlining studies that span multiple sites. ${ }^{23}$ A developer must apply to obtain the necessary approval from these committees by supplying the detailed plan (protocol) of the experiment to be carried out and, preferably, advance permission from all of the participating centers. LRECs are guided to consider the following general criteria and any other criteria specific to the experiment: ${ }^{24}$

1. Has the scientific merit of the proposal been properly assessed?

2. How will the health of the research subjects be affected?

3. Are there possible hazards and, if so, adequate facilities to deal with them?

4. What degree of discomfort or distress is foreseen?

5. Is the investigation adequately supervised and is the supervisor responsible for the project adequately qualified and experienced?

6. What monetary or other inducements are being offered to the NHS body, doctors, researchers, subjects or anyone else involved?

7. Are there proper procedures for obtaining consent from the subjects or where necessary their parents or guardians?

8. Has an appropriate information sheet for the subjects been prepared?

An illustration of ethics committee workings, for FDA-supported research can be found via the US Office of Human Research Protections. ${ }^{25}$ This explains the role of Institutional Review Boards (IRBs), the equivalent of RECs in the US.

Research ethics has a considerable impact on the methods by which evidence on the efficacy of a medical device can be obtained. Protection of human subjects and patients is paramount, and stringent legislation covering all human subjects is accompanied by special rules to protect specific groups, for example children, prisoners and cognitive disabled persons. Furthermore, in recent years special research ethics rules and guidelines have been devised for experiments involving genetics, human and animal tissues and organs. These guidelines may become more relevant to device designers as the number of drug-device combination products proliferates. Animal experimentation is receiving renewed ethical consideration in 
many countries as a result of greater attention to animal welfare issues, the question of efficacy of animal models and cost, and especially due to a more concerted adoption of the principles of the 3R's replacement, reduction and refinement of animal use. In Australia, this has become most sophisticated by means of the Animal Ethics Committees (AECs) that have been introduced into animal welfare legislation through a revised Australian code of practice for the care and use of animals for scientific purposes. ${ }^{26}$ The AECs act as decision-making bodies a similar manner to RECs. In the UK, a government-funded Centre for the $3 R^{\prime}$ 's has recently been established whose stated aim is the ultimate replacement of animal use. ${ }^{27}$ Further information about research ethics for both humans and animals can be obtained via the Online Ethics Center website. ${ }^{28}$

In addition to RECS there are a number of other types of ethics committees. In the UK for example, Clinical Ethics Committees (CECs) provide advice and support on ethical issues arising from clinical practice and patient care within health care organizations. ${ }^{29}$ Although these committees are not decision-making bodies like RECs, they may influence aspects of healthcare such as hospital procedures and equity of access, which may be quite relevant to a technology developer. Finally, ethics in business is experiencing a growth in concern within the medical device sector, which is relevant to the collaboration of companies with healthcare professionals (HCPs). For example, the US medical devices trade association AdvaMed has recently adopted a voluntary Code of Ethics to facilitate ethical interactions with HCPs who may 'purchase, lease, recommend, use, arrange for the purchase or lease of, or prescribe Members' medical technology products in the U.S.'. ${ }^{30}$

\subsubsection{Advances in clinical trials for devices}

The 'gold standard' for clinical trials is the Randomized Controlled Trial (RCT) that was developed for evaluating drug treatments but is now used for the full range of clinical procedures. However, there are some problems associated with RCTs for medical devices because of the differences between devices and drugs as shown previously in Table 1. In a useful illustration of this, Lilford et al. have outlined some of the issues involved in surgical trials, which often involve devices, showing how application of the RCT can be problematic. ${ }^{31}$ These issues include ethics of intervention versus non-intervention, the problem of blinding of participants (surgeons, patients and hospital staff), surgeon- and technique-related variables, timing of trials, patient preferences, entry criteria, appropriate outcome measures, and a number of statistical considerations. As a result, less powerful clinical studies, such as cohort studies and casestudies, are quite prevalent in the literature for medical devices. However, the value of studies lower down in the hierarchy of evidence can be difficult to assess from the HTA perspective. Lilford et al. argue strongly for randomization and in a separate paper propose tracker trials for comparing fast-changing technologies like devices. ${ }^{32}$ Tracker trials include the set of contemporary examples of treatments employed by clinicians but allow for the addition of new devices and treatments into the study as the trial progresses. This is to help ensure early adoption if a new procedure is found to be superior, as well as for removal of a procedure if it shown to be performing poorly or if it has been superseded by technological developments. These are still randomized trials, but they do not have preset and rigid protocols and are ongoing studies rather than the one-off event that characterizes an RCT. New developments of this kind may become influential in the future of medical device assessments. 


\section{Routes for medical devices innovation}

Having covered some of the nuts and bolts of medical device development and their accompanying regulations and standards in the previous two sections, we are now in a position to look at the overall innovation process.

\subsection{New product development processes}

New Product Development (NPD) methods typically break the innovation process down into a series of stages with associated decision-making, called stage-gate systems. ${ }^{33}$ Such processes are aimed at helping to reduce development time and improving quality through standardization of practice and subsequent capture of best practice. Rochford and Rudelius have studied medical devices in this context, comparing 'new-to-the-world' products and product modifications using a 12-stage process. ${ }^{34}$

Members of the author's Multidisciplinary Assessment of Technology Centre for Healthcare (MATCH) have recently completed a consultation with our industrial partners that has resulted in a simplified 4stage generic process for medical devices, giving a snap-shot of the current approach to product development in the industry ${ }^{35,36}$. This process is summarized in Figure 1. Much of the progression of stages will be familiar to project managers and product developers. However, there are some points for special note. Firstly, there will be more stages in practice. The 4-stage model is intended to be a high-level representation of the decision-making steps involved. Secondly, as a result of the regulatory processes that must be undertaken, the first three stages involve a great deal of planning before detailed product design can be attempted, after which strict design controls must be in place to track and document all modifications. This is quite typical of safety-critical systems. These controls and the validation processes that close the iterative loops during Stage 3 have received attention by others in terms of guidance to the medical device industry (e.g. the Cambridge Engineering Design Centre's Design for Validation approach). ${ }^{37}$

(INSERT FIGURE 1 ABOUT HERE - find figure after references)

A notable omission in documented current practice is specific planning for HTA and reimbursement as mentioned earlier in the chapter. This is being addressed in MATCH by the promotion of health economics modeling ${ }^{38}$ which would benefit from its earliest adoption in Stage 1, with subsequent refinement at other stages, such as during clinical trials. ${ }^{39}$ The other main omissions are the environmental and sustainability strategies that will have to be put in place as the newer regulations are introduced. These will require the industry to go beyond dealing with retirement of obsolete devices and towards advance planning for disposal at the point when detailed requirements are drawn up and design decisions 
involving choices of materials and energy use are made. Sourcing, procurement, reporting, servicing and repair are all areas that will be affected by such legislation. ${ }^{40}$

A further development areas is the approach to stakeholder requirements, especially in the area of user needs capture to ensure devices are fit-for-purpose. As we have seen this is another area that is moving from guidance towards regulation, and will benefit from review throughout the lifecycle, suggesting the introduction of more formal human factors methods into the concept design phase of Stage 2 and specific planning for usability testing in Stage 3.

\subsection{Special considerations for pervasive medical device development}

For devices involving data, which are inherent to pervasive computing in healthcare, the communication and security issues mentioned earlier will need specific planning for since they will impact on design choices, user testing and post-market surveillance. Since regulations and standards are in continual development it will be most beneficial for pervasive healthcare designers to engage with the bodies concerned with these. Interaction with patient groups will help provide insights into public concerns about protection of personal records.

Since many pervasive healthcare devices are likely to be used in the home, this raises obvious concerns in the area of maintenance and disposal/recycling as mentioned above. Comparison with the experiences of deploying equipment for disabled users by healthcare providers would be useful in this respect. As well as those supplied by professionals, many pervasive healthcare devices are likely to be purchased over-thecounter, and this will raise further issues such as ensuring adequate training.

Finally, the best combined methods to use for user needs capture of pervasive healthcare devices in conjunction with the ICT systems they interact with is still an open question that would benefit from further research. ${ }^{41}$ 


\section{Conclusions}

The environment of the pervasive healthcare innovator is a multidisciplinary one, bringing together the roles of the clinical designer/engineer and ICT specialist. It is hoped that this chapter has provided an insight into the processes of healthcare technology development. On the device side especially, there is a need for understanding the regulatory processes within the product life-cycle plus other aspects which might normally be outside a designers remit, such as reimbursement and HTA processes. Bringing this knowledge into the design process should help to improve decision-making in medical device innovation.

In a large medical devices organization, there will most likely be expert individuals and departments in some of the specific areas outlined in this chapter such as clinical trials and regulations so it will not be necessary for designers to have detailed knowledge of these. Of course, this is the benefit of a multidisciplinary team. For a small company or university research group, however, it is more likely that individuals will need to become multidisciplinary to some extent.

Medical device regulations and standards are presently going through great changes and the web has become an important medium for accessing the latest information and advice, highlighted by the large number of online references in this chapter. Official sources of information on regulations and extensive guidance include the Medical Devices Directives ${ }^{10}$ and MEDDEV ${ }^{12}$ in the European Union, Device Advice ${ }^{9}$ in the USA, and via the websites of the other members of the Global Harmonization Task Force ${ }^{8}$. Industry supporting websites and magazines such as Medical Devicelink ${ }^{42}$, Medical Device Technology ${ }^{43}$ and publications of professional bodies such as the IEEE Engineering in Medicine and Biology Society ${ }^{44}$, are further useful sources of advice and information for medical technology developers.

Pervasive computing is an exciting field that should contribute in addressing and solving many of issues involved in developing new healthcare technologies and bringing them to patients.

\section{Acknowledgements}

The author acknowledges support of this work through the MATCH Programme (EPSRC Grant GR/S29874/01), although the views expressed are entirely his. Many thanks are due to MATCH research staff Shirley Davey, Julie Eatock, Sukhvinder Johal for their valuable comments on the initial draft of the manuscript, and to the editors and reviewers for their subsequent remarks. 


\section{References}

[1] Booth, A. The ScHARR Guide to Evidence-based Practice, ScHARR (School of Health and Related Research) Occasional Paper No. 97/2, University of Sheffield, 1997, ISBN 1-900752-03-4. See also, http://www.nettingtheevidence.org.uk/ website (accessed 21 March 2005).

[2] Baxter, M. Product Design: a practical guide to systematic methods of new product development, Chapman and Hall, London, 1995, ISBN 0-412-632306.

[3] Bromley. A., Bensenane, J., Riley, K., Sullivan, K., Mazzei, L., Guglieri, A., Arrizabalaga-Moreno, F., Alfonsel, M., and Rozynski, E. Gaining Reimbursement for Medical Devices and Diagnostics, Clinica Report CBS924, Strategic Management Reports Series, PJB Publications Ltd., March 2004.

[4] Papatheofanis, F.J. Estimating cost in health economic analyses, IEEE Engineering in Medicine and Biology magazine, 22(5), 14-15, 2003.

[5] NHS HTA Programme website, National Coordinating Centre for Health Technology Assessment (NCCHTA), UK, http://www.ncchta.org/ (accessed 10 March 2005).

[6] Centre for Reviews \& Dissemination (CRD) website. University of York, UK, http://www.york.ac.uk/inst/crd/ (accessed 15 March 2005).

[7] Taylor, R. National Institute for Clinical Excellence (NICE). HTA rhyme and reason? International Journal of Technology Assessment in Health Care, 18(2), 166-70, 2002.

[8] Global Harmonization Task Force (GHTF) website. http://www.ghtf.org/ (accessed 14 March 2005).

[9] Device Advice website. Center for Devices and Radiological Health, Food and Drug Administration (FDA), USA, http://www.fda.gov/cdrh/devadvice/ (accessed 15 March 2005).

[10] Europa - Enterprise - Medical Devices website.

http://europa.eu.int/comm/enterprise/medical_devices/ (accessed 12 March 2005).

[11] Higson, G.R. Medical Device Safety: the regulation of medical devices for public health and safety, IOP Publishing, Bristol, 2002, ISBN 0750307684.

[12] MEDDEV. Guidelines relating to medical devices directives, Europa - Enterprise - Medical Devices, http://europa.eu.int/comm/enterprise/medical_devices/meddev/ (accessed 12 March 2005).

[13] Davey, S., Anderson, J., and Meenan, B. An overview of current classification systems for healthcare devices and their limitations, Multidisciplinary Assessment of Technology Centre for Healthcare (MATCH), Deliverable 2, P1 D2 V2.0 051025, May 2005. Available from http://www.match.ac.uk/

[14] World Health Organization. Medical device regulations: global overview and guiding principles. WHO, Geneva: ] World Health Organization,2003, ISBN 94-4-154618.

[15] International Standards Organisation (ISO) website, http://www.iso.org/ (accessed 15 March 2005). 
[16] Fries, R.C. Reliable design of medical devices, Marcel Dekker Inc., New York, 1997, ISBN 0-8247-98430 .

[17] Cohen, T. Medical and Information Technologies converge: the impact on clinical engineering, IEEE Engineering in Medicine and Biology magazine, 23(3), 59-65, 2004.

[18] Integrating the Healthcare Enterprise (IHE) website, http://www.ihe.net/ (accessed 23 March 2005).

[19] Reid, P.P., Compton, W.D., Grosman, J.H., and Fanjiang G. (eds.). Building a Better Delivery System: A New Engineering/Health Care Partnership, National Academic of Engineering and Institute of Medicine, The National Academies Press, http://www.nap.edu/books/030909643X/html (accessed 10 Dec 2005).

[20] Health Insurance Portability and Accountability Act (HIPAA). Office for Civil Rights, US Department of Health and Human Services website, http://www.hhs.gov/ocr/hipaa/ (accessed 23 March 2005).

[21] Grimes, S.L. Security: a new clinical engineering paradigm, IEEE Engineering in Medicine and Biology magazine, 23(4), 80-82, 2004

[22] Boutrand, J.P. When to conduct a clinical trial, Medical device technology (MDT), Octomedia Ltd., October 2004:28-31. Available from http://www.medicaldevicesonline.com/

[23] Central Office for Research Ethics Committees (COREC) website, National Patient Safety Agency, National Health Service, UK, http://www.corec.org.uk/ (accessed 29 March 2005).

[24] Local Research Ethics Committees, HSG(91)5, 1991. Department of Health, Heywood, Lancs., UK. Available online from DH Publications Library, http://www.dh.gov.uk/PublicationsAndStatistics/Publications/PublicationsLibrary/fs/en/ (accessed 21 March 2005).

[25] Code of Federal Regulations, Protection of Human Subjects, 45 CFR Part 46, 2001, Office of Human Research Protections, US Department of Health and Human Services website, http://www.hhs.gov/ohrp/humansubjects/guidance/45cfr46.htm (accessed 14 March 2005).

[26] Animal Ethics webpage, National Health and Medical Research Council, Ethical Issues, Australia, http://www.nhmrc.gov.au/issues/animalethics.htm (accessed 14 March 2005).

[27] National Centre for the Replacement, Refinement and Reduction of Animals in Research (NC3R) website, UK, http://www.nc3rs.org.uk/ (accessed 14 March 2005).

[28] Online Ethics Center (OEC), Reference Materials on Research Ethics, http://onlineethics.org/reseth/reference.html (accessed 14 March 2005).

[29] Clinical Ethics Network (CEN) website, http://www.ethics-network.org.uk/ (accessed 21 March 2005).

[30] Code of Ethics for Interactions with Health Care Professionals, Advanced Medical Technology Association (Advamed), http://www.advamed.org/publicdocs/coe.html (accessed 14 March 2005).

[31] Lilford, R., Braunholtz, D., Harris, J., and Gill, T. Trials in surgery, Br. J. Surgery, 91, 6-16, 2004. 
[32] Lilford RJ, Braunholtz DA, Greenhalgh R, Edwards SJL, Trials and fast changing technologies: the case for tracker studies, Br. Med. J., 320, 43-46, 2000.

[33] Cooper RG. Stage-gate systems: a new tool for managing new products, Business Horizons, 33(3), 4454, 1990.

[34] Rochford, L. and Rudelius, W. Stages and success in the medical products industry, Industrial Marketing Management, 26, 67-84, 1997.

[35] Dixon, D., Eatock, J., Meenan, B., Young, T., and Anderson, J. MATCH guide on product development processes in the medical device industry, Multidisciplinary Assessment of Technology Centre for Healthcare (MATCH), Deliverable 9, P2 D9 V2.0 051025, May 2005. Available from http://www.match.ac.uk/

[36] Eatock, J., Dixon, D., Meenan, B., Young, T., Anderson, J. Report on medical device product development processes : some illustrative examples, Multidisciplinary Assessment of Technology Centre for Healthcare (MATCH), Deliverable 5, P2 D5 V2 051025, April 2005. Available from http://www.match.ac.uk/

[37] CEDC (2005), Cambridge Engineering Design Centre, Healthcare webpage, University of Cambridge, http://www-edc.eng.cam.ac.uk/healthcare/ (accessed 22 March 2005).

[38] Health Economics Research Group (HERG) website, Brunel University, UK, http://www.brunel.ac.uk/about/acad/herg/ (accessed 22 March 2005).

[39] Johnston, K., Buxton, M.J., Jones, D.R., and Fitzpatrick, R. Assessing the costs of healthcare technologies in clinical trials. Health Technology Assessment ; 3(6), 1999.

[40] Wong, C., On the horizon: new rules for medical devices:

Preparing for new environmental regulations and product compliance, Agile SME Solutions, News, August 23, 2004, http://sme.agile.com/news/2004/biosci-082304.pdf (accessed 22 March 2005).

[41] Craven, M.P. and Martin, J.L. How does the healthcare industry involve users in medical device development? - Pointers for UbiHealth, UbiHealth 2004: The 3rd International Workshop on Ubiquitous Computing for Pervasive Healthcare Applications, at UbiComp 2004, the Sixth International Conference on Ubiquitous Computing, Nottingham, England, 7-10 September 2004.

http://www.pervasivehealthcare.com/ubicomp2004/papers/ (accessed 22 March 2005).

[42] Medical Devicelink, the platform website for the medical device industry, http://www.devicelink.com/ (accessed 31 March 2005).

[43] Medical Device Technology (MDT), Medical devices online, http://www.medicaldevicesonline.com/ (accessed 15 March 2005).

[44] Institute of Electrical and Electronic Engineers (IEEE), Engineering in Medicine and Biology Society, Engineering in Medicine and Biology Magazine, http://www.ieee.org/organizations/pubs/magazines/emb.htm (accessed 31 March 2005). 


\section{Tables and figures}

Table 1 Comparison of medical devices and drugs

\begin{tabular}{|c|c|c|}
\hline & Devices & Drug \\
\hline Principal Action & $\begin{array}{l}\text { Other than pharmacological, immunological or metabolic } \\
\text { means } \\
\text { Often mechanical, electrical or materials engineering } \\
\text { based }\end{array}$ & $\begin{array}{l}\text { Pharmacological, immunological or metabolic } \\
\text { means } \\
\text { Chemical based }\end{array}$ \\
\hline Product Life Cycle & $\begin{array}{l}\text { Constantly evolving, incremental modification } \\
\text { Often short life cycle }\end{array}$ & $\begin{array}{l}\text { Unchanging compound } \\
\text { Long life cycle }\end{array}$ \\
\hline $\begin{array}{l}\text { Clinical } \\
\text { Evaluation }\end{array}$ & $\begin{array}{l}\text { Lower requirement for clinical investigation (class } \\
\text { dependent) } \\
\text { Difficult to blind (to find a placebo), crossover common } \\
\text { Difficult to stabilize for conventional RCT } \\
\text { Multiple end users }\end{array}$ & $\begin{array}{l}\text { More formalized evaluation and extensive clinical } \\
\text { investigation } \\
\text { Easy to blind with a placebo, crossover rare } \\
\text { Use conventional Randomized Controlled Trials } \\
\text { Usually one end user }\end{array}$ \\
\hline Use issues & $\begin{array}{l}\text { Results vary with operator skill } \\
\text { Wide range of over-the-counter availability } \\
\text { Often require intensive training to use } \\
\text { Complications decrease with use }\end{array}$ & $\begin{array}{l}\text { Results unrelated to physician skill } \\
\text { Majority prescribed } \\
\text { Low training requirement } \\
\text { Complications increase with use }\end{array}$ \\
\hline Diversity & A few large companies and many small companies (SMEs) & Mainly large multinational companies \\
\hline Costs & $\begin{array}{l}\text { Varying overheads with slow return } \\
\text { High distribution cost } \\
\text { High maintenance and disposal/recycling costs }\end{array}$ & $\begin{array}{l}\text { High overheads, but quicker return } \\
\text { Low distribution cost } \\
\text { Low disposal costs }\end{array}$ \\
\hline
\end{tabular}


Table 2. Examples of product classifications arising from MDD rules, collated from guidance document MEDDEV 2.4/1 - rev. 8 PART 2: July $2001^{12}$

\begin{tabular}{|c|c|}
\hline Device Class & Examples \\
\hline I, low risk & $\begin{array}{l}\text { Active diagnostic devices intended to illuminate or view the patient's body in the visible spectrum e.g. examination lights, } \\
\text { surgical microscopes. Dental curing lights. } \\
\text { Active diagnostic devices intended for thermography; devices for recording, processing or viewing of diagnostic images. } \\
\text { Devices intended in general for external patient support e.g. hospital beds, patient hoists, walking aids, wheelchairs, } \\
\text { stretchers, dental patient chairs. } \\
\text { Incontinence pads, non-sterile dressings, plaster of Paris, corrective glasses, non-invasive electrodes (for EEG or ECG), image } \\
\text { intensifying screens, cups and spoons, syringes without needles, dentistry mirrors, gloves, reusable scalpels, saw blades, } \\
\text { tubing for transient use. }\end{array}$ \\
\hline $\begin{array}{l}\text { Ila, medium } \\
\text { risk }\end{array}$ & $\begin{array}{l}\text { Active therapeutic devices intended to administer or exchange energy, unless doing so in potentially hazardous way: } \\
\text { Electrical, magnetic, electromagnetic - Muscle stimulators and external bone growth stimulators, TENS devices and eye, } \\
\text { electromagnets, electrical acupuncture; Thermal - Cryosurgery equipment, heat exchangers, except the types described } \\
\text { below; Mechanical - Powered dermatomes, powered drills and dental hand pieces; Light - Phototherapy for skin treatment } \\
\text { and for neonatal care; Sound - Hearing aids; Ultrasound: Equipment for physiotherapy. } \\
\text { Many active devices intended for diagnosis e.g. imaging devices (MRI, evoked response, diagnostic ultrasound, Gamma } \\
\text { cameras, PET, SPECT), ECG, EEG, electronic thermometers, stethoscopes \& blood pressure measuring equipment. Also, X-ray } \\
\text { films, photostimulable phosphor plates. } \\
\text { Active devices intended to administer and/or remove medicines, body liquids or other substances to or from the body, } \\
\text { unless potentially hazardous e.g. Suction equipment, feeding pumps, Jet injectors for vaccination, non-dose critical nebulae's } \\
\text { Channels for active drug delivery and blood, syringes for infusion pumps, devices for temporary storage of transplant organs } \\
\text { or long-term storage of bodily materials, filtering machines \& centrifuges (mechanical separation). Most tubing for short } \\
\text { term use e.g. urinary catheters, tracheal tubes, stents. Wound dressings managing the micro-environment e.g. polymer film. } \\
\text { Dental prostheses. } \\
\text { Many surgically invasive devices intended for transient use e.g. needles used for suturing, needles of syringes, single use } \\
\text { scalpels/blades, drill bits connected to active devices, trial implants. } \\
\text { Devices intended specifically to be used for disinfecting medical devices, except contact lens solution. }\end{array}$ \\
\hline Ilb, elevated & $\begin{array}{l}\text { Active therapeutic devices intended to administer or exchange energy, doing so in potentially hazardous way: Kinetic energy } \\
\text { - lung ventilators; Thermal energy - incubators, warming blankets, blood warmers, heat exchangers; Electrical energy - high- } \\
\text { frequency electrosurgical generators, electrocautery equipment and electrodes, external pacemakers, external defibrillators, } \\
\text { electroconvulsive therapy equipment; Coherent light - surgical lasers; Ultrasound - lithotriptors, surgical ultrasound devices; } \\
\text { lonizing radiation - radioactive sources for afterloading therapy, therapeutic cyclotrons, linear accelerators, therapeutic X-ray } \\
\text { sources. Also, active devices intended to control and monitor the performance of active therapeutical devices in Class Ilb e.g. } \\
\text { external feedback systems. Also, diagnostic X-ray sources. } \\
\text { Intensive care monitoring and alarm devices (for e.g. blood pressure, temperature, oxygen saturation), biological sensors, } \\
\text { blood gas analyzers used in open heart surgery, cardioscopes and apnea monitors, including apnea monitors in home care. } \\
\text { Active devices intended to administer and/or remove medicines, body liquids or other substances to or from the body, } \\
\text { unless potentially hazardous - Infusion pumps, ventilators, anesthesia machines, anesthetic vaporizers, dialysis equipment, } \\
\text { blood pumps for heart-lung machines, hyperbaric chambers, pressure regulators for medical gases, medical gas mixers, } \\
\text { moisture exchangers in breathing circuits if used on unconscious or non-spontaneously breathing patients, dose-critical } \\
\text { nebulae's. } \\
\text { Catheters incorporating sealed radioisotopes, excluding the central circulatory system (CCS), tubing for long term use e.g. }\end{array}$ \\
\hline
\end{tabular}




\begin{tabular}{|l|l|}
\hline & $\begin{array}{l}\text { urethral stents, Wound dressings with secondary healing properties. } \\
\text { Many implantable devices e.g. prosthetic joint replacements, non-implantable devices used for contraception or the } \\
\text { prevention of the transmission of sexually transmitted diseases e.g. condoms, diaphragms. Dose-critical medicine applicators } \\
\text { e.g. insulin pens, surgically invasive adhesives, contact lens solutions. }\end{array}$ \\
\hline III, high risk & $\begin{array}{l}\text { Cardiovascular catheters e.g. angioplasty balloon catheters incl. guidewires, dedicated disposable cardiovascular surgical } \\
\text { instruments. Catheters incorporating sealed radioisotopes for central circulatory system (CCS). }\end{array}$ \\
$\begin{array}{l}\text { Implantable devices to be used in direct contact with the heart, the CCS or the central nervous system (CNS) e.g. heart } \\
\text { valves, spinal stents, CNS electrodes. Implantable contraceptives e.g. intrauterine devices. Bioactive implantable devices e.g. } \\
\text { absorbable sutures and biological adhesives. } \\
\text { Rechargeable non-active drug delivery systems. } \\
\text { All devices incorporating a medicinal product as an integral part with an action ancillary to that of the devices: Antibiotic } \\
\text { bone cements, condoms with spermicide, heparin coated catheters, endodontic materials with antibiotics, some ophthalmic } \\
\text { irrigation solutions, dressings incorporating an antimicrobial agent to provide ancillary action on the wound. }\end{array}$ \\
\hline
\end{tabular}


Figure 1. A four-stage innovation process for medical device development (Adapted from Dixon et al. ${ }^{35}$ and Eatock et al. ${ }^{36}$ With permission.)

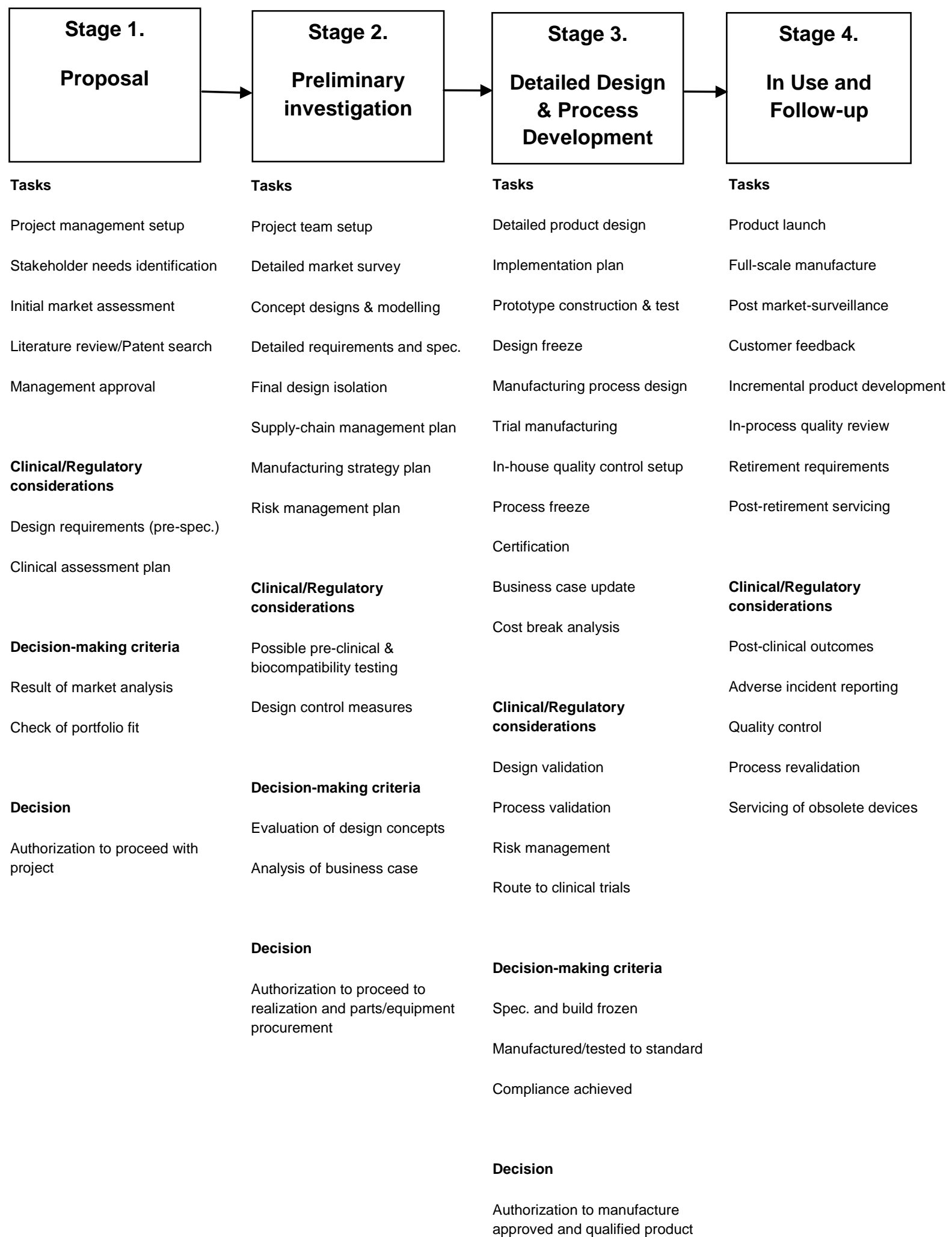

